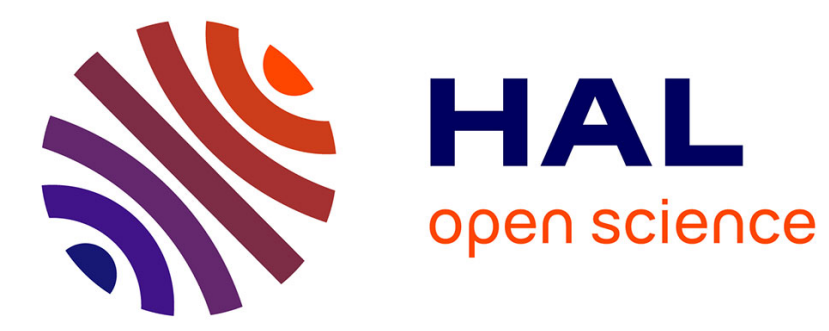

\title{
Effects of inter-subband couplings on bound and extended states of terahertz cascade structures
}

C. Ndebeka-Bandou, F. Carosella, Gérald Bastard

\section{To cite this version:}

C. Ndebeka-Bandou, F. Carosella, Gérald Bastard. Effects of inter-subband couplings on bound and extended states of terahertz cascade structures. Journal of Applied Physics, 2016, 119 (9), pp.095703. 10.1063/1.4942848 . hal-01310920

\section{HAL Id: hal-01310920 \\ https://hal.sorbonne-universite.fr/hal-01310920}

Submitted on 3 May 2016

HAL is a multi-disciplinary open access archive for the deposit and dissemination of scientific research documents, whether they are published or not. The documents may come from teaching and research institutions in France or abroad, or from public or private research centers.
L'archive ouverte pluridisciplinaire HAL, est destinée au dépôt et à la diffusion de documents scientifiques de niveau recherche, publiés ou non, émanant des établissements d'enseignement et de recherche français ou étrangers, des laboratoires publics ou privés. 


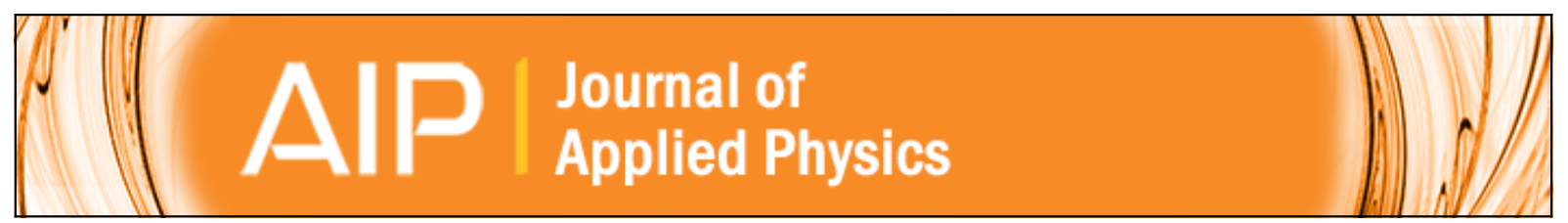

\section{Effects of inter-subband couplings on bound and extended states of terahertz cascade structures}

C. Ndebeka-Bandou, F. Carosella, and G. Bastard

Citation: Journal of Applied Physics 119, 095703 (2016); doi: 10.1063/1.4942848

View online: http://dx.doi.org/10.1063/1.4942848

View Table of Contents: http://scitation.aip.org/content/aip/journal/jap/119/9?ver=pdfcov

Published by the AIP Publishing

\section{Articles you may be interested in}

Strong coupling and stimulated emission in single parabolic quantum well microcavity for terahertz cascade Appl. Phys. Lett. 107, 101101 (2015); 10.1063/1.4930165

Rigorous coherent-structure theory for falling liquid films: Viscous dispersion effects on bound-state formation and self-organization

Phys. Fluids 23, 044104 (2011); 10.1063/1.3572061

Bound states in coupled guides. II. Three dimensions

J. Math. Phys. 45, 1380 (2004); 10.1063/1.1675932

Bound states in coupled guides. I. Two dimensions

J. Math. Phys. 45, 1359 (2004); 10.1063/1.1675931

The Dynamics of Bound States of Topological Defects in Extended Spatially Periodic Structures AIP Conf. Proc. 622, 359 (2002); 10.1063/1.1487554

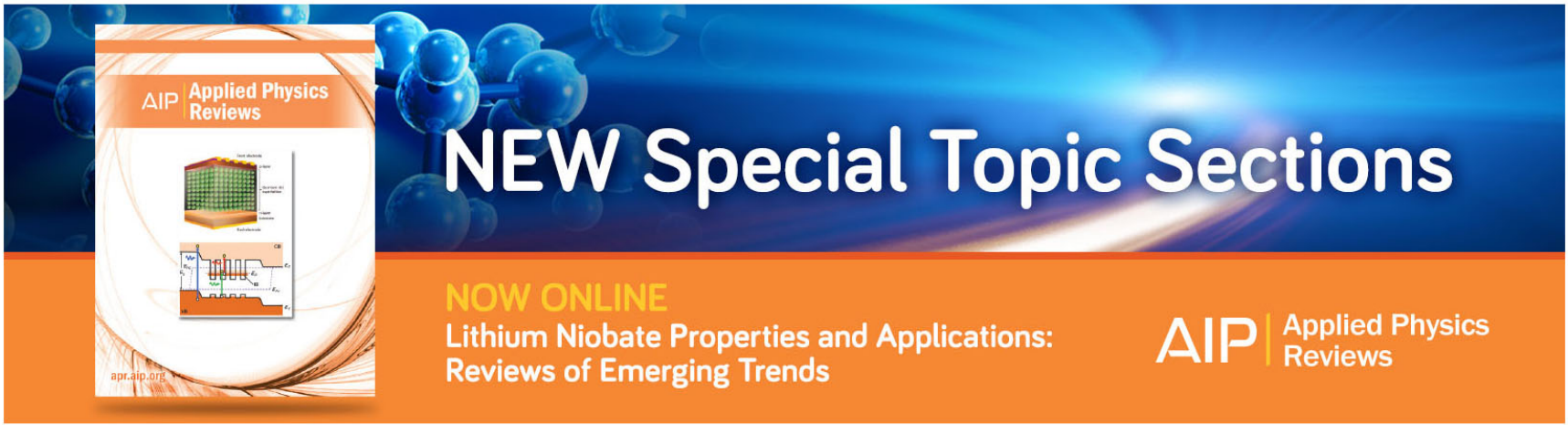




\title{
Effects of inter-subband couplings on bound and extended states of terahertz cascade structures
}

\author{
C. Ndebeka-Bandou, ${ }^{1}$ F. Carosella, ${ }^{2}$ and G. Bastard ${ }^{2,3}$ \\ ${ }^{1}$ Institute for Quantum Electronics, ETH Zürich, Auguste-Piccard-Hof 1, 8093 Zürich, Switzerland \\ ${ }^{2}$ Laboratoire Pierre Aigrain, Ecole Normale Supérieure, CNRS (UMR 8551), Université P. et M. Curie, \\ Université Paris Diderot, 24 rue Lhomond, F-75005 Paris, France \\ ${ }^{3}$ Photonics Institute, Vienna University of Technology, Gusshausstrasse 27-29, 1040 Vienna, Austria
}

(Received 29 October 2015; accepted 13 February 2016; published online 1 March 2016)

\begin{abstract}
We theoretically analyze the effect of inter-subband couplings on the eigenstates of a terahertz heterostructure in the presence of coulombic donors. We find that the inter-subband electron-donor interaction causes a drastic reorganization of the eigenenergies and a strong admixture between the subbands that decisively influences the optical absorption spectrum of the structure. These results illustrate the importance of the inter-subband couplings in the formulation of the eigenstates of imperfect terahertz heterostructures. (C) 2016 AIP Publishing LLC.
\end{abstract}

[http://dx.doi.org/10.1063/1.4942848]

\section{INTRODUCTION}

While Quantum Cascade Lasers (QCLs) emitting in the mid-infrared are now well-established devices operating at room temperature, ${ }^{1-3}$ such is not the case for terahertz $(\mathrm{THz})$ emitting QCLs. ${ }^{4-7}$ There is one obvious reason which is the energy spacing between the upper and lower laser levels that becomes comparable or smaller than the thermal energy $k_{B T}$ and its concomitant difficulties to achieve a population inversion between these two levels. ${ }^{8}$ A less obvious reason is the matching between this energy spacing and typical coulombic impurity effects (for instance, the binding energy of bulk GaAs-like materials is $\approx 5 \mathrm{meV}$, which is $\approx 1.2 \mathrm{THz}$ ). This coincidence between the relevant energy spacings and the typical coulombic effects suggests that the usual carrier dynamics in these $\mathrm{THz}$ emitting materials might be quite different from the ones that prevails in mid-infrared emitting structures. In the latter, the carrier gets scattered very efficiently in a given subband and only occasionally undergoes an inter-subband transfer. In $\mathrm{THz}$ emitting structures, the inter-subband scattering times shorten and become comparable to the intra-subband ones.

In the present paper, we wish to examine the consequences of the growing efficiency of inter-subband scattering effects on the modification of the impurity-related optical properties. To be specific, we shall confine ourselves to Double Quantum Well structures (DQWs), where the pair of lower lying states is well separated from the other states. An example is the $\mathrm{GaAs} / \mathrm{Ga}_{0.85} \mathrm{Al}_{0.15} \mathrm{As} \mathrm{DQW}$ structure with layer thicknesses 17/2.5/16.5 nm (see Fig. 1). Measured from the GaAs conduction band edge (the bottom of the wells in the DQW), the two lower lying states of interest have an energy $E_{1}=10.22 \mathrm{meV}$ and $E_{2}=13.49 \mathrm{meV}$. These two states are dipole-coupled when the electric field of the electromagnetic wave is parallel to the growth axis ( $z$ polarization). Such a DQW could be useful for $\mathrm{THz}$ emission or detection..$^{9,10}$ Our first task will be to ascertain the magnitude of the inter-subband coupling on the bound state(s) created by a coulombic donor screened by a $2 \mathrm{D}$ electron gas with a fixed areal concentration of $n=n_{\text {free }}=2 \times 10^{10} \mathrm{~cm}^{-2}$. These carriers are supplied to the DQW by injection and removed from the DQW by extraction. The injection and extraction currents are equal in magnitude in the steady state. We shall assume thermal equilibrium at a temperature $T$. For a given $T$, the fixed concentration of mobile electrons imposes the chemical potential (see the Appendix). The latter, in turn fixes the concentration of electrons bound to donor impurities. Section II briefly summarizes the model we use for electronic state as well as the shape of the screened coulombic bound state. Section III will display the pronounced subband coupling effects in the built up of the bound state as well as the optical response that involves the coulombic bound states. After dealing with bound states, we shall discuss the effects of subband coupling on delocalized states in Section IV.

\section{TWO SUBBAND MODEL}

The one electron Hamiltonian we shall use is

$$
\begin{aligned}
H & =p_{z} \frac{1}{2 m^{*}(z)} p_{z}+\frac{p_{x}^{2}+p_{y}^{2}}{2 m^{*}(z)}+V_{\mathrm{conf}}(z)+V_{\mathrm{imp}}(\vec{r}) \\
& =H_{0}+V_{\mathrm{imp}}(\vec{r})
\end{aligned}
$$

where $m^{*}(z)$ is the position-dependent effective mass and $V_{\text {conf }}(z)$ is the DQW conduction band profile depicted in Fig.1. The eigenstates of $H_{0}$ factorize

$$
\psi(\vec{r})=\chi_{n}(z) \frac{1}{\sqrt{S}} \exp (i \vec{k} \cdot \vec{\rho})
$$

$E_{1}$ and $E_{2}$ are the two lower lying subband edges, the lower eigenstates of $H_{0}$ at $k_{x}=k_{y}=0$. In GaAs/Ga(Al)As heterostructures, the effective masses in the wells and barriers are close from each other. Hence, it is an excellent approximation (perturbative estimate) to write the $\vec{k}$-dependent eigenvalues as $^{11}$ 


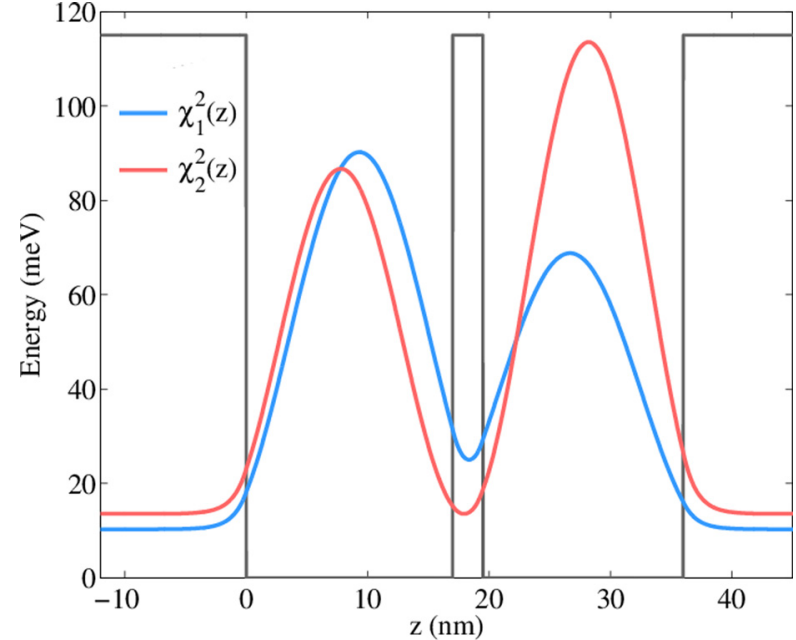

FIG. 1. Conduction band edge profile of the DQW and plots of the $\chi_{1}^{2}(z)$ and $\chi_{2}^{2}(z)$ probability densities versus $z$.

$$
\begin{gathered}
E_{n}(\vec{k}) \approx E_{n}+\frac{\hbar^{2}\left(k_{x}^{2}+k_{y}^{2}\right)}{2 m_{n}^{*}}, \\
\frac{1}{m_{n}^{*}}=\frac{1}{m_{\text {well }}^{*}} P_{n}^{\text {well }}+\frac{1}{m_{\text {barrier }}^{*}} P_{n}^{\text {barrier }},
\end{gathered}
$$

where $P_{n}^{\text {well }}$ and $P_{n}^{\text {barrier }}$ are the probability to find the electron of the $n$th subband in the well material and in the barrier material, respectively. The structures we shall numerically analyze will contain $1,2, \ldots$ impurities. This corresponds to an areal concentration of $n_{\mathrm{imp}}=(1,2, \ldots) \times 2.5 \times 10^{9} \mathrm{~cm}^{-2}$.

These are light dopings and when several impurities are considered they are far away from each other and without any interaction. A donor impurity located at $\vec{R}_{i}=\left(\vec{\rho}_{i}, z_{d}\right)$ creates a screened coulombic potential due to the presence of mobile electrons. ${ }^{12}$ Following Nelander and Wacker, ${ }^{13}$ we adopt a Debye type of screening

$$
\begin{gathered}
V_{\text {imp }}(\vec{r})=-\frac{e^{2}}{4 \pi \varepsilon_{0} \varepsilon_{r}\left|\vec{r}-\vec{R}_{i}\right|} \exp \left(-\frac{\left|\vec{r}-\vec{R}_{i}\right|}{\lambda_{D}}\right), \\
\frac{1}{\lambda_{D}}=\sqrt{\frac{e^{2} n_{3 \mathrm{D}}}{\varepsilon_{0} \varepsilon_{r} k_{B} T}},
\end{gathered}
$$

where $n_{3 \mathrm{D}}=n / L$ with $n$ being the $2 \mathrm{D}$ areal concentration of mobile electrons and $L$ being the length of the DQW structure. We note that our assumption of fixed $n$ (external supply of electrons) implies a decreasing screening efficiency with increasing temperature. Note that in the case of a DQW not supplied with carriers, the concentration of mobile electrons would have to adjust to the concentration of thermally depopulated donors. This would result in a decreasing $n$ with decreasing $T$ and therefore a decreasing screening efficiency with decreasing temperature. For low carrier concentration and temperatures considered in the paper, the electron gas is non degenerate.

Taking into account the two lowest subbands of the DQW, the solutions of (1) are searched in the form

$$
\psi_{\nu}(\vec{r})=\chi_{1}(z) f_{1 \nu}(\vec{\rho})+\chi_{2}(z) f_{2 \nu}(\vec{\rho}) .
$$

The functions $f_{1}$ and $f_{2}$ are the solutions of the system

$$
\begin{aligned}
& \left(\begin{array}{cc}
H_{\text {intra }}^{(1)} & \left\langle\chi_{1}\left|V_{\text {imp }}\right| \chi_{2}\right\rangle \\
\left\langle\chi_{2}\left|V_{\text {imp }}\right| \chi_{1}\right\rangle & H_{\text {intra }}^{(2)}
\end{array}\right)\left(\begin{array}{l}
f_{1} \\
f_{2}
\end{array}\right)=\varepsilon\left(\begin{array}{l}
f_{1} \\
f_{2}
\end{array}\right), \\
& H_{\text {intra }}^{(i)}=E_{i}-\frac{\hbar^{2} \nabla^{2}}{2 m_{i}^{*}}+\left\langle\chi_{i}\left|V_{\text {imp }}\right| \chi_{i}\right\rangle ; \quad i=1,2,
\end{aligned}
$$

where $V_{\text {imp }}(\vec{r})=\sum_{l=1}^{N_{d}} V_{\text {imp }}\left(\vec{r}-\vec{R}_{l}\right)$ is actually a sum over all the impurity sites $\vec{R}_{l}$. To solve (9), we expand $f_{1}$ and $f_{2}$ on the eigenfunctions of the intra-subband Hamiltonians. We note that these eigenstates contain a continuum as well as bound states $^{14-16}$ since $V_{\text {imp }}$ is the sum of screened coulombic terms with long screening lengths due to the low carrier concentration. For simplicity, we shall assume that each impurity binds only one state below each subband edge; the generalization to several bound states per impurity would not be a difficulty

$$
\begin{aligned}
& \left|f_{1}\right\rangle=\sum_{l=1}^{N_{d}} c_{l \text { bound }}^{(1)}\left|\varphi_{\text {loc }, l}\right\rangle+\sum_{i} c_{\nu_{i}}^{(1)}\left|\varphi_{\nu_{i}}\right\rangle, \\
& \left|f_{2}\right\rangle=\sum_{l=1}^{N_{d}} c_{l \text { bound }}^{(2)}\left|\xi_{\text {loc }, l}\right\rangle+\sum_{j} c_{\mu_{j}}^{(2)}\left|\xi_{\mu_{j}}\right\rangle .
\end{aligned}
$$

In the presence of inter-subband coupling, the allowed energies will be the solutions of the linear system

$$
\begin{aligned}
& c_{l_{0} \text { bound }}^{(1)}\left(\varepsilon_{b}^{(1)}-\varepsilon\right)+c_{l_{0} \text { bound }}^{(2)}\left\langle\varphi_{\text {loc }, l_{0}}\left|\left\langle\chi_{1}\left|V_{\text {imp }}^{l_{0}}\right| \chi_{2}\right\rangle\right| \xi_{\text {loc }, l_{0}}\right\rangle \\
& +\sum_{j} c_{\mu_{j}}^{(2)}\left\langle\varphi_{\text {loc }, l_{0}}\left|\left\langle\chi_{1}\left|V_{\text {imp }}^{l_{0}}\right| \chi_{2}\right\rangle\right| \xi_{\mu_{j}}\right\rangle=0, \\
& c_{\nu_{i}}^{(1)}\left(\varepsilon_{\nu_{i}}^{(1)}-\varepsilon\right)+\sum_{l=1}^{N_{d}} c_{l \text { bound }}^{(2)}\left\langle\varphi_{\nu_{i}}\left|\left\langle\chi_{1}\left|V_{\text {imp }}^{l}\right| \chi_{2}\right\rangle\right| \xi_{\text {loc }, l}\right\rangle \\
& +\sum_{j, l} c_{\mu_{j}}^{(2)}\left\langle\varphi_{\nu_{i}}\left|\left\langle\chi_{1}\left|V_{\text {imp }}^{l}\right| \chi_{2}\right\rangle\right| \xi_{\mu_{j}}\right\rangle=0, \\
& c_{l_{0} \text { bound }}^{(2)}\left(\varepsilon_{b}^{(2)}-\varepsilon\right)+c_{l_{0} \text { bound }}^{(1)}\left\langle\xi_{\text {loc }, l_{0}}\left|\left\langle\chi_{2}\left|V_{\text {imp }}^{l_{0}}\right| \chi_{1}\right\rangle\right| \varphi_{\text {loc }, l_{0}}\right\rangle \\
& +\sum_{i} c_{\nu_{i}}^{(1)}\left\langle\xi_{\text {loc }, l_{0}}\left|\left\langle\chi_{2}\left|V_{\text {imp }}^{l_{0}}\right| \chi_{1}\right\rangle\right| \varphi_{\nu_{i}}\right\rangle=0, \\
& c_{\mu_{j}}^{(2)}\left(\varepsilon_{\mu_{j}}^{(2)}-\varepsilon\right)+\sum_{l=1}^{N_{d}} c_{l \text { bound }}^{(1)}\left\langle\xi_{\mu_{j}}\left|\left\langle\chi_{2}\left|V_{\text {imp }}^{l}\right| \chi_{1}\right\rangle\right| \varphi_{\text {loc }, l}\right\rangle \\
& +\sum_{i, l} c_{\nu_{i}}^{(1)}\left\langle\xi_{\mu}\left|\left\langle\chi_{2}\left|V_{\text {imp }}^{l}\right| \chi_{1}\right\rangle\right| \varphi_{\nu_{i}}\right\rangle=0,
\end{aligned}
$$

where in (13) and (15), the impurity dilution has been used to neglect the inter-subband coupling between bound states associated with distinct impurities. In Eqs. (13)-(16), $\varepsilon_{b}^{(1)}$ and $\varepsilon_{\nu_{i}}\left(\varepsilon_{b}^{(2)}\right.$ and $\left.\varepsilon_{\mu_{j}}\right)$ are the eigenvalues of $H_{\text {intra }}^{(1)}\left(H_{\text {intra }}^{(2)}\right)$, respectively, for the bound state and the continuum states. We shall analyze below the structure of these equations for the bound states and extended states.

In the presence of impurities, there are no exact solutions of (9)-(16). We have numerically solved the $\{\mathrm{DQW}+$ impurity Hamiltonian by expanding the eigenstates of (9) 
on the basis $\left|\chi_{1}, \vec{k}_{m n}\right\rangle,\left|\chi_{2}, \vec{k}_{m n}\right\rangle$ where $\left|\vec{k}_{m n}\right\rangle$ are the plane waves made periodic on a $200 \times 200 \mathrm{~nm}^{2}$ surface. The dimension of the Hilbert space is 200 states for the numerical computations with inter-subband couplings (i.e., $200 \times 200$ matrices for the computations with inter-subband couplings and $100 \times 100$ matrices for intra-subband couplings only). The largest $k$ values retained in the calculations are such that the in-plane kinetic energy is $15 \mathrm{meV}$, i.e., both $E_{1}$ and $E_{2}$ dispersions extend $15 \mathrm{meV}$ above their edges.

Once the eigenstates and eigenvalues of (1) are known, the optical properties can be calculated. The absorption coefficient $\alpha(\omega)$ is proportional to ${ }^{17}$

$$
\alpha(\omega) \propto \sum_{\mu, \nu}\left[f\left(\varepsilon_{\mu}\right)-f\left(\varepsilon_{\nu}\right)\right]\left|\left\langle\psi_{\nu}\left|p_{z}\right| \psi_{\mu}\right\rangle\right|^{2} \delta\left(\varepsilon_{\nu}-\varepsilon_{\mu}-\hbar \omega\right) .
$$

\section{EFFECT OF SUBBAND COUPLING ON BOUND STATES}

For a single impurity, we consider the position dependence of the impurity binding energy against the impurity position along the growth direction. Three main cases will be discussed (see Fig. 2): when the impurity is placed in the injection barrier $\left(z_{d}=-8 \mathrm{~nm}\right)$, at the maximum of $\chi_{1}^{2}(z)\left(z_{d}=9.3 \mathrm{~nm}\right)$ or at the maximum of $\chi_{2}^{2}(z)\left(z_{d}=28.2\right.$ $\mathrm{nm})$.

The impurity potential admits intra-subband and intersubband matrix elements. We have previously shown that in the case of large $E_{2}-E_{1}$ splittings compared to typical inter-subband impurity matrix elements, the intra-subband contributions are dominant and the inter-subband ones merely blur the bound states attached to $E_{2}$ that results from the diagonalization of the intra- $E_{2}$ subband Hamiltonian. ${ }^{18,19}$

We follow the same approach and solve first the intrasubband Hamiltonian. For an impurity placed at the maximum of $\chi_{2}^{2}(z)$ (respectively, $\chi_{1}^{2}(z)$ ) probability density, we find a bound state $\varepsilon_{b}^{(1)}$ below $E_{1}$ at $9 \mathrm{meV}(8.35 \mathrm{meV})$ and a

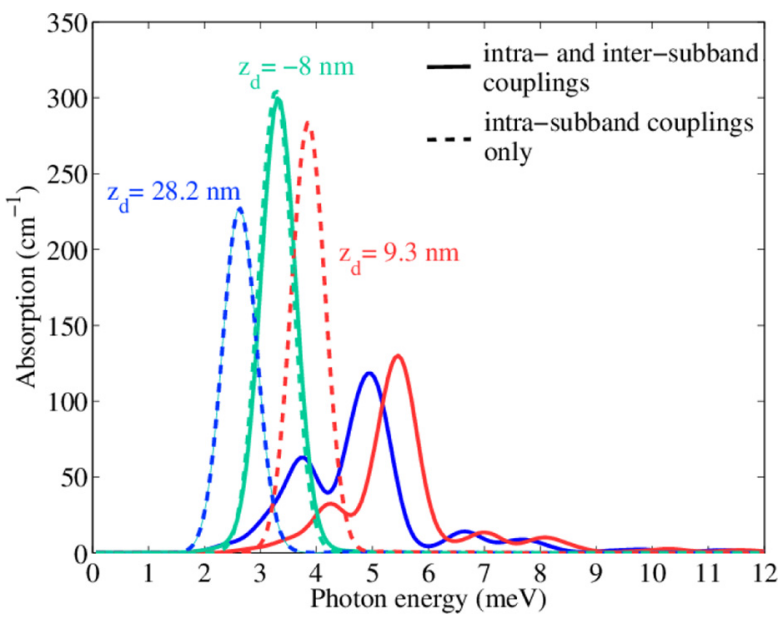

FIG. 2. Absorption coefficient versus photon energy for three different impurity positions: $z_{d}=9.3 \mathrm{~nm}$ and $z_{d}=28.2 \mathrm{~nm}$ correspond to the maximum of $\chi_{1}^{2}(z)$ and $\chi_{2}^{2}(z)$, respectively, $z_{d}=-8 \mathrm{~nm}$ corresponds to a position in the injection barrier of the DQW. Dashed lines: intra-subband couplings only. Solid lines: full calculations including inter-subband couplings. $T=3 \mathrm{~K}$. bound state $\varepsilon_{b}^{(2)}$ below $E_{2}$ at $11.66 \mathrm{meV}(12.33 \mathrm{meV})$. In the decoupled subband scheme at low temperature $(T=3 \mathrm{~K})$, only $\varepsilon_{b}^{(1)}$ is occupied and we find in the $z$ polarization a $\varepsilon_{b}^{(1)}$ $\rightarrow \varepsilon_{b}^{(2)}$ bound-to-bound transition at $2.65 \mathrm{meV}(3.88 \mathrm{meV})$ reminiscent of the $1 \mathrm{~s} \rightarrow 2 \mathrm{p}_{z}$ transition of hydrogenic atoms. When $T$ increases to $30 \mathrm{~K}$ (see Fig. 3), the lower extended states of the $E_{1}$ subband become populated and a second peak develops. It corresponds to the "regular" inter-subband transition centered at $E_{2}-E_{1}=3.27 \mathrm{meV}$. Increasing further $T$ leads to a disappearance of the bound-to-bound transition and to a decrease of the inter-subband absorption strength due to the thermal activation of the stimulated emission.

Turning on the inter-subband coupling leads to a drastic reorganization of the eigenenergies as well as to important changes of the absorption spectra. The $\varepsilon_{b}^{(1)}$ level undergoes a red shift and, in the presence of inter-subband couplings, is now found at $7.79 \mathrm{meV}(7.39 \mathrm{meV})$ when the impurity position is at the maximum of $\chi_{2}^{2}(z)\left(\chi_{1}^{2}(z)\right)$, see Fig. 4. It is impossible to securely follow what happens for $\varepsilon_{b}^{(2)}$ since the state (if it exists) is now a resonance superimposed to the $E_{1}$ continuum. However, the coupling to the continuum states often results in a broadening of this state while only a marginally small shift of its position takes place. Such a small shift is not expected for the bound-bound coupling. Hence, in a first attempt, we neglect any coupling between the bound states and the continuums. Thus, we describe the bound state with subband mixing by a two-level model. We readily find that the two bound (or quasi-bound) states are now found at the energies

$$
\begin{gathered}
\varepsilon_{ \pm}=\frac{\varepsilon_{b}^{(1)}+\varepsilon_{b}^{(2)}}{2} \pm \sqrt{\left(\frac{\varepsilon_{b}^{(2)}-\varepsilon_{b}^{(1)}}{2}\right)^{2}+\lambda^{2},} \\
\lambda=\left|\left\langle\varphi_{\text {loc }, l_{0}}\left|\left\langle\chi_{1}\left|V_{\text {imp }}^{l_{0}}\right| \chi_{2}\right\rangle\right| \xi_{\text {loc }, l_{0}}\right\rangle\right|,
\end{gathered}
$$

where $\lambda$ is the inter-subband coupling matrix element between the two bound states of the intra-subband Hamiltonian for the

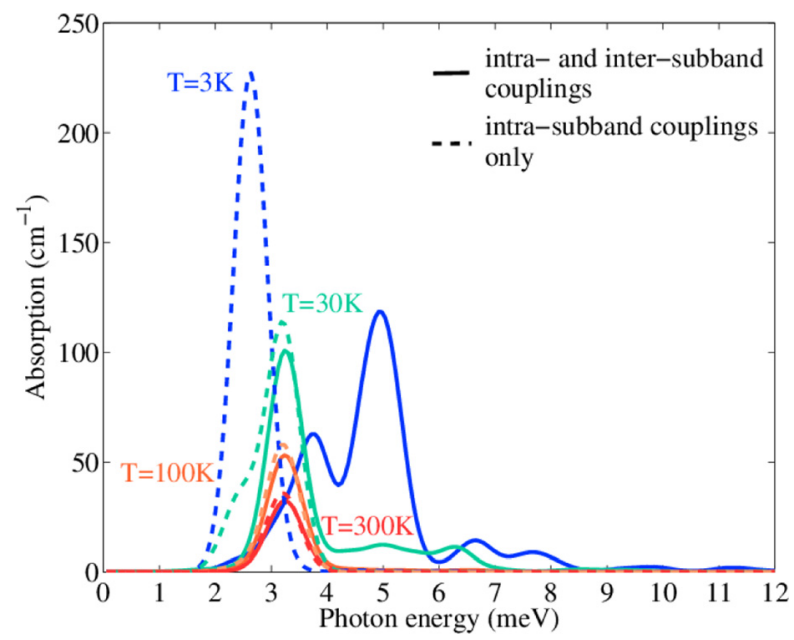

FIG. 3. Absorption coefficient versus photon energy at various temperatures. Dashed lines: intra-subband couplings only. Solid lines: full calculations including inter-subband couplings. $z_{d}=28.2 \mathrm{~nm}$. 


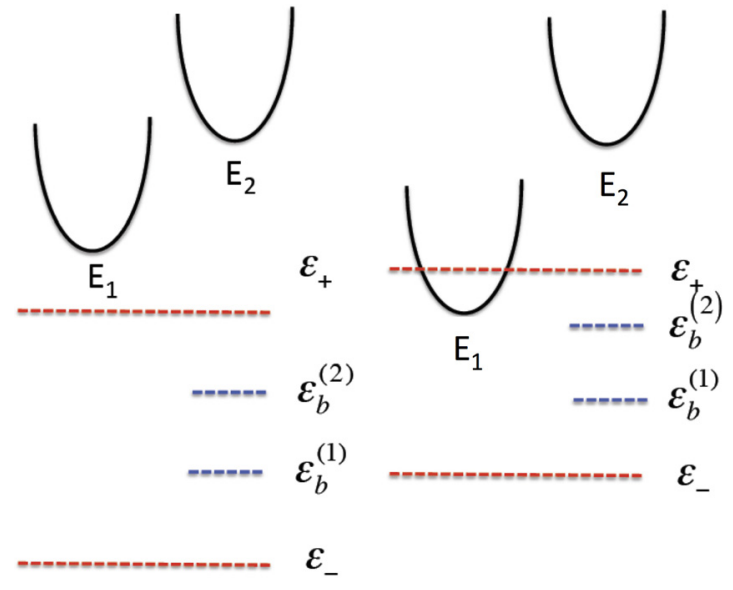

FIG. 4. Qualitative sketches of the bound states created by a screened coulombic donor in the presence of two subbands. Dashed blue lines: result of the intra-subband diagonalization. Red dashed lines: result including intersubband couplings. In the left panel, the inter-subband coupling is weak enough to make that the states attached to $E_{1}$ and $E_{2}$ remain bound, i.e., with an energy smaller than $E_{1}$. Note that (right panel) there exist situations where the upper bound state of the intra-subband Hamiltonian is pushed in the ( $E_{1}$ or $E_{1}$ and $\left.E_{2}\right)$ continuum(s) due to the inter-subband coupling. In practice, this can only happen when the subband separation is comparable to the binding energy that results from intra-subband diagonalization.

same impurity. Since we know $\varepsilon_{b}^{(1)}, \varepsilon_{b}^{(2)}$, and $\varepsilon_{-}$from the numerical diagonalization, we immediately extract from (18) that $\lambda \approx 2.2 \mathrm{meV}$ for both impurity locations. This has the important consequence that in the presence of inter-subband couplings the state bound to $E_{2}$ is actually pushed above $E_{1}$ $\left(\varepsilon_{+}>E_{1}\right)$ and becomes a resonance degenerate with the $E_{1}$ continuum (see Fig. 4, right panel). This changes the nature of the eigenstates of the imperfect $\mathrm{THz}$ designed heterostructures. The existence of a virtual bound state is an intrinsic quantum mechanical property that is not affected by temperature. The effects on the transport properties associated with the virtual bound state will be as affective at low as at high temperature.

The inter-subband mixing drastically changes the absorption spectra, because it allows a lot more optical transitions with a non-negligible oscillator strength. At the lowest temperature $(T=3 \mathrm{~K})$, there are two main absorption peaks near $3.8 \mathrm{meV}(4.1 \mathrm{meV})$ and $5 \mathrm{meV}(5.5 \mathrm{meV})$ when the impurity is located at the maximum of the $\chi_{2}^{2}(z)\left(\chi_{1}^{2}(z)\right)$ probability density (see Fig. 2). Note that when the impurity is in the injection barrier there is only a single peak found near $E_{2}-E_{1}$. The most important peak seen in Fig. 3 is remarkable because it occurs at an energy very close from the $\varepsilon_{+}-\varepsilon_{-}$energy difference $(5.08 \mathrm{meV}$ and $5.80 \mathrm{meV}$, respectively, from Eqs. (18) and (19)) and therefore corresponds to the "resonance" transition of the coulombic impurity (quasi $1 s \rightarrow 2 p_{z}$ ). Note however, that the final (pseudo $2 p_{z}$ ) state is degenerate with the $E_{1}$ continuum. The $3.8 \mathrm{meV}(4.1 \mathrm{meV})$ feature corresponds to the "ionization" transition from $\varepsilon_{-}$to the $E_{1}$ continuum. It is blue shifted from the onset $E_{1}-\varepsilon_{-}$ $(2.43 \mathrm{meV}$ and $2.83 \mathrm{meV}$, respectively) by a quantity of the order of the binding energy and reminiscent of what is found

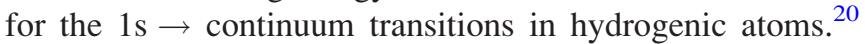
The occurrence of this peak is also remarkable because it points out the existence of a strong admixture between the subbands (without subband mixing this ionization transition would be forbidden since $\left\langle\chi_{1}\left|p_{z}\right| \chi_{1}\right\rangle=0$ ). We note that a similar peak corresponding to the $\varepsilon_{-} \rightarrow E_{2}$ transition shows up near $6.6 \mathrm{meV}(7 \mathrm{meV})$. These $6.6 \mathrm{meV}(7 \mathrm{meV})$ peaks are in fact roughly separated from the $3.8 \mathrm{meV}(4.1 \mathrm{meV})$ peaks by $E_{2}-E_{1}$. Fig. 3 shows $\alpha(\omega)$ at different temperatures. When $T$ increases all the impurity-related features decrease to the benefit of the "regular" inter-subband transition near $E_{2}-E_{1}$.

\section{EFFECT OF SUBBAND COUPLING ON THE EXTENDED STATES}

A possible way to assert the importance of subband mixing consists in evaluating for each state $\psi_{\nu}$ the square projections $P_{i}^{(\nu)}=\int d^{2} \rho\left|\left\langle\chi_{i} \mid \psi_{\nu}\right\rangle\right|^{2}, i=1,2$. There is of course $P_{1}^{(\nu)}+P_{2}^{(\nu)}=1$. In the case of large subband separation, the departure from an initial state in one subband due to intersubband coupling can be handled perturbatively by the Fermi golden rule. ${ }^{21}$ In the case of very close subbands, such a simple approach has to be improved. Fig. 5 shows $P_{1}^{(\nu)}, P_{2}^{(\nu)}$ versus the energy of the state when the DQW has been doped with 1 donor in a $200 \times 200 \mathrm{~nm}^{2}$ at $z_{d}=28.2$ $\mathrm{nm}$. We see that the low lying part of the spectrum (i.e., from below $E_{1}$ to few meVs above $E_{1}$ ) displays very strong subband mixing effects. This starts with the bound state where $P_{1} \approx 0.77$ (in agreement with the two level model (18)). Note also the mixing occurring near $E_{2}$. It arises from the presence of the resonant state $\varepsilon_{+}$in the vicinity of the subband edge $E_{2}$. Fig. 6 shows a plot similar to Fig. 5 but where the impurity potential has been divided by 10 . No bound state below $E_{1}$ is found due to the weakness of the donor potential. Correlatively, the subband mixing displayed in Fig. 6 is much weaker than the one shown in Fig. 5. A very similar trend is observed in Fig. 7 that corresponds to an impurity placed in the injection barrier. These trends are explained by the existence of a subband-admixed resonant state between $E_{1}$ and $E_{2}$. The eigenstates of the DQW show a significant subband admixture for energies between $E_{1}$ and

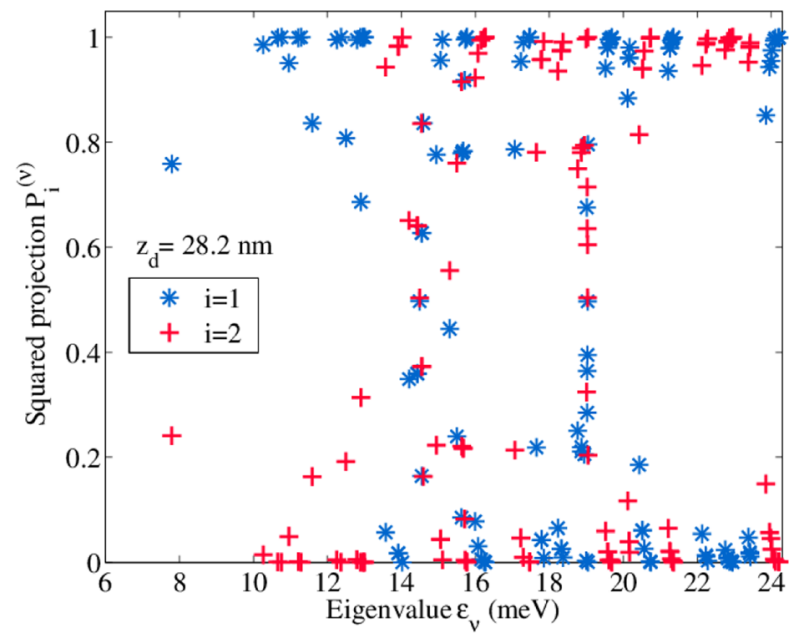

FIG. 5. Squared projections $P_{1}$ and $P_{2}$ versus energy for a DQW doped by one donor located at $z_{d}=28.2 \mathrm{~nm} . \varepsilon_{b}^{1}=9 \mathrm{meV}, \varepsilon_{b}^{2}=11.66 \mathrm{meV}$ and $\varepsilon_{-}=$ $7.8 \mathrm{meV} . T=3 \mathrm{~K}$ 


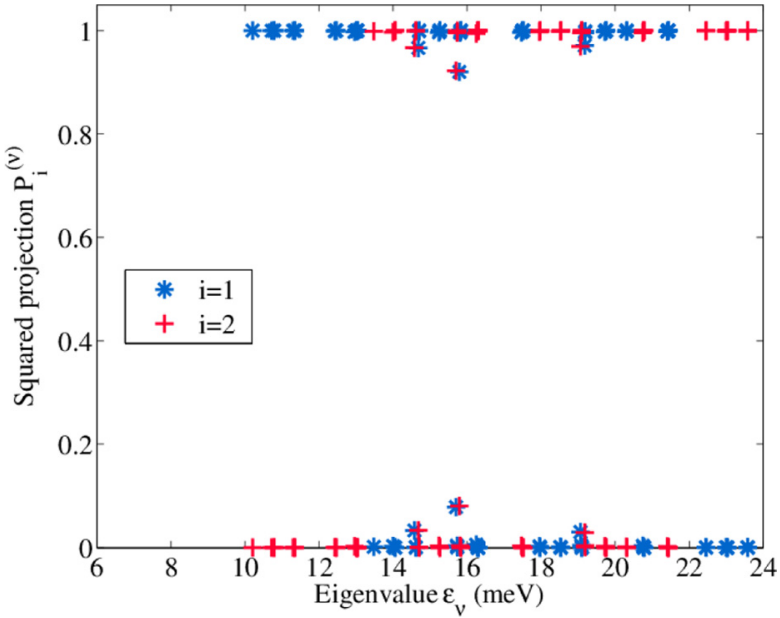

FIG. 6. Squared projections $P_{1}$ and $P_{2}$ versus energy for a DQW doped by one donor located at $z_{d}=28.2 \mathrm{~nm}$. The strength of the impurity potential has been divided by 10. $\varepsilon_{b}^{1}=\varepsilon_{-}=10.2 \mathrm{meV}\left(\approx E_{1}\right)$ and $\varepsilon_{b}^{2}=13.47 \mathrm{meV}$ $\left(\approx E_{2}\right) . T=3 \mathrm{~K}$.

$E_{2}$ because the subband mixing has lifted up the bound state $\varepsilon_{b}^{(2)}$ above the subband edge $E_{1}$. Suppose we restrict the expansion of (11) and (12) to

$$
\begin{gathered}
\left|f_{1}\right\rangle=c_{\text {bound }}^{(1)}\left|\varphi_{\text {loc }, \mu_{0}}\right\rangle+\sum_{\nu_{i}} c_{\nu_{i}}^{(1)}\left|\varphi_{\nu_{i}}\right\rangle, \\
\left|f_{2}\right\rangle=c_{\text {bound }}^{(2)}\left|\xi_{\text {loc }, \mu_{0}}\right\rangle,
\end{gathered}
$$

i.e., we remove the intra-subband $E_{2}$ continuum. Then, the diagonalization of the subband coupling between the remaining states is particularly simple since

$$
\begin{gathered}
\left(\begin{array}{cccccc}
\varepsilon_{b}^{(1)} & \lambda & 0 & 0 & \ldots & 0 \\
\lambda^{*} & \varepsilon_{b}^{(2)} & \beta_{\mu_{0}}^{\left(\nu_{1}\right)} & \beta_{\mu_{0}}^{\left(\nu_{2}\right)} & \ldots & \beta_{\mu_{0}}^{\left(\nu_{N}\right)} \\
0 & \beta_{\mu_{0}}^{*\left(\nu_{1}\right)} & \varepsilon_{\nu_{1}} & 0 & \ldots & 0 \\
0 & \beta_{\mu_{0}}^{*\left(\nu_{2}\right)} & 0 & \varepsilon_{\nu_{2}} & \ldots & 0 \\
\ldots & \cdots & \ldots & \ldots & \ldots & 0 \\
0 & \beta_{\mu_{0}}^{*\left(\nu_{N}\right)} & 0 & 0 & 0 & \varepsilon_{\nu_{N}}
\end{array}\right)\left(\begin{array}{c}
c_{\text {bound }}^{(1)} \\
c_{\text {bound }}^{(2)} \\
c_{\nu_{1}}^{(1)} \\
c_{\nu_{2}}^{(1)} \\
\vdots \\
c_{\nu_{\mathrm{N}}}^{(1)}
\end{array}\right) \\
=\varepsilon\left(\begin{array}{c}
c_{\text {bound }}^{(1)} \\
c_{\text {bound }}^{(2)} \\
c_{\nu_{1}}^{(1)} \\
c_{\nu_{2}}^{(1)} \\
\vdots \\
\beta_{\mu_{0}}^{\left(\nu_{j}\right)}= \\
c_{\nu_{\mathrm{N}}}^{(1)}
\end{array}\right) \\
\left\langle\xi_{\text {loc }, \mu_{0}}\left|\left\langle\chi_{2}\left|V_{\mathrm{imp}}^{\mu_{0}}\right| \chi_{1}\right\rangle\right| \varphi_{\nu_{i}}\right\rangle
\end{gathered}
$$

and therefore,

$$
\varepsilon_{b}^{(2)}-\varepsilon+\frac{|\lambda|^{2}}{\varepsilon-\varepsilon_{b}^{(1)}}+\sum_{i} \frac{\left|\beta_{\mu_{0}}^{\left(\nu_{i}\right)}\right|^{2}}{\varepsilon-\varepsilon_{\nu_{i}}}=0 .
$$

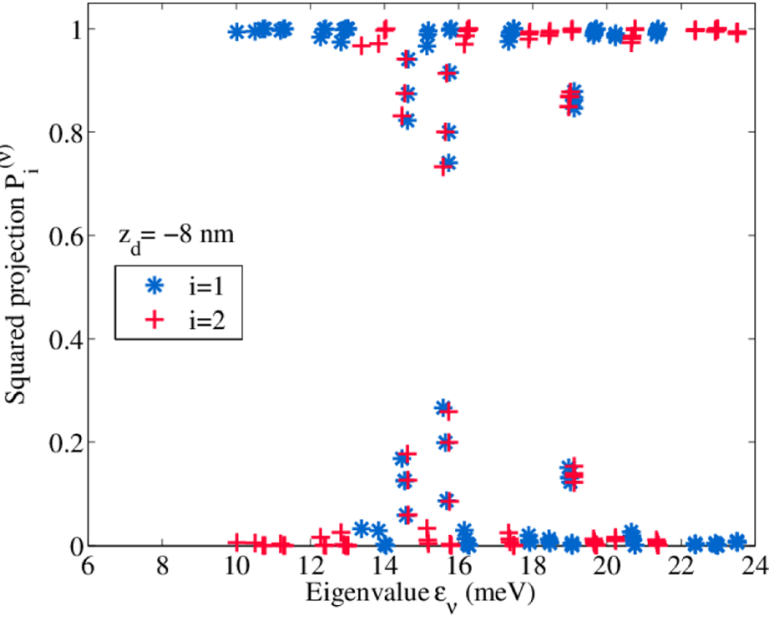

FIG. 7. Squared projections $P_{1}$ and $P_{2}$ versus energy for a DQW doped by one donor located in the injection barrier. $\varepsilon_{b}^{1}=10.03 \mathrm{meV}, \varepsilon_{b}^{2}=13.34 \mathrm{meV}$, and $\varepsilon_{-}=10.01 \mathrm{meV} . T=3 \mathrm{~K}$

We recall that the rest of the inter-subband coupling is non resonant since it involves $E_{2}$ extended states that lay higher in energy than the ones in (20)-(22). We see readily from (22) that all the states derived from $E_{1}$ by diagonalizing the intra-subband terms now acquire a non zero $E_{2}$ character due to the presence of the $E_{2}$ bound state

$$
c_{\nu_{i}}^{(1)}=\frac{\beta_{\mu_{0}}^{\left(\nu_{i}\right) *}}{\varepsilon-\varepsilon_{\nu_{i}}} c_{\text {bound }}^{(2)} .
$$

Fig. 8 shows the squared projections $P_{1}$ and $P_{2}$ versus the eigenvalue when four donors stay at $z_{d}=28.2 \mathrm{~nm}$. As expected, the subband mixing has increased significantly despite the fact that $n_{\mathrm{imp}} \approx 10^{10} \mathrm{~cm}^{-2}$. Actual $\mathrm{THz}$ devices should therefore be characterized by a pronounced subband mixing for electron states that are statistically relevant. Instead, when $E_{2}-E_{1}$ becomes much larger than the donor binding energy, there should exist very little subband mixing except near the $E_{2}$ edge.

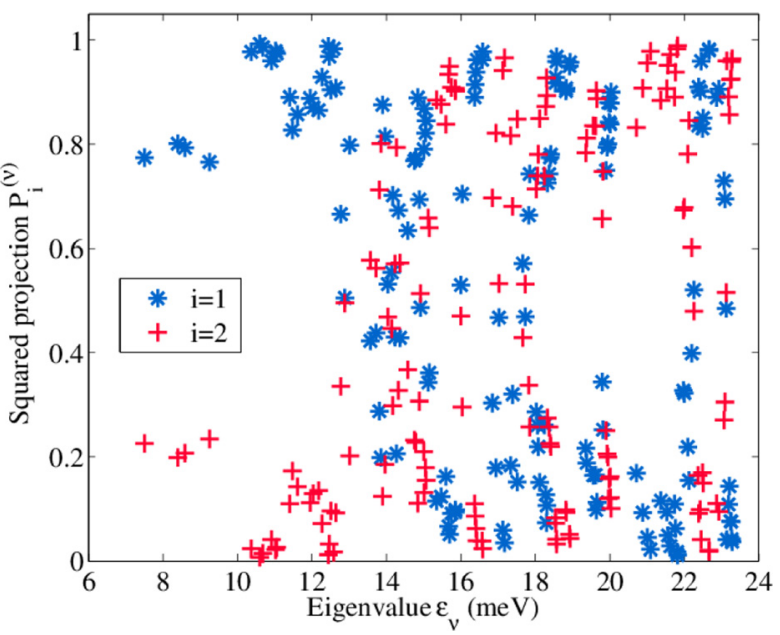

FIG. 8. Squared projections $P_{1}$ and $P_{2}$ versus energy for a DQW doped by four donors located at $z_{d}=28.2 \mathrm{~nm} . T=3 \mathrm{~K}$. 


\section{CONCLUSION}

We have shown that even at a very low doping concentration $\left(2.5 \times 10^{9} \mathrm{~cm}^{-2}\right)$, there exists a substantial mixing between the eigenstates of the $E_{1}$ and the $E_{2}$ subbands if the subband separation is comparable to the impurity binding energy. The electronic spectrum is completely reorganized by impurity scattering over a $10 \mathrm{meV}$ range from the subband edges. This leads to a drastic change of the subband absorption lineshape. Moreover, because of the presence of bound states, the absorption lineshape is strongly temperature dependent. Consequences on the transport data remain to be evaluated.

\section{ACKNOWLEDGMENTS}

The LPA-ENS is UMR 8551 of the Centre National de la Recherche Scientifique (CNRS). The work at LPA-ENS has been supported by the Paris Diderot and Pierre et Marie Curie Universities. The authors whish to acknowledge Professor J. Faist, Dr. Ohtani, Professor K. Unterrainer, and Professor G. Strasser for enlightening discussions. C.-N.B. wants to thank the support from the ERC under the project MUSiC.

\section{APPENDIX: CONCENTRATION OF NEUTRAL DONORS AND CHEMICAL POTENTIAL VERSUS $T$}

Under the assumption of thermal equilibrium, there are two different physical situations that lead to different values of the chemical potential and of the concentration of neutral impurities at a given temperature $T$ : the first situation corresponds to a DQW that does not exchange any particle with the surrounding. In this case, the areal concentration of mobile electrons $n_{\text {free }}$ adjusts to the concentration of ionized donors $n_{\text {ion. }}$. The value of the chemical potential $\mu$ results from this charge neutrality. Once $\mu$ is known, the concentration of electrons bound to the donor sites then follows from (A2) and (A3).

The second situation corresponds to a DQW that is supplied/depleted with electrons due to a permanent current flowing in the structure. In this case, the concentration of mobile electrons is fixed. The chemical potential adjusts to this value at all $T$, and the concentration of neutral donors follows from (A2) and (A3). There is always

$$
\exp \left(\frac{\pi \hbar^{2} n_{\text {free }}}{m^{*} k_{B} T}\right)-1=e^{\beta\left(\mu-E_{1}\right)},
$$

$$
\begin{gathered}
n_{\text {bound }}=\frac{n_{\mathrm{imp}}}{1+\frac{1}{2} e^{\beta\left(E_{1}-R^{*}-\mu\right)}}, \\
n_{\mathrm{ion}}=\frac{n_{\mathrm{imp}}}{1+2 e^{-\beta\left(E_{1}-R^{*}-\mu\right)}},
\end{gathered}
$$

where $\beta=\left(k_{B} T\right)^{-1}$ and $R^{*}$ is the donor binding energy.

To draw the absorption coefficient versus $T$, we have assumed that the second situation holds. Thus,

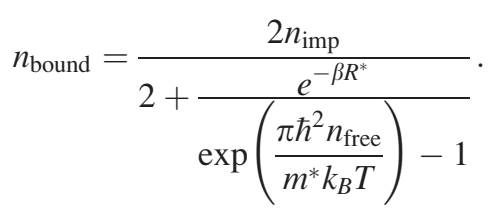

${ }^{1}$ Y. Yao, A. J. Hoffman, and C. F. Gmachl, Nat. Photonics 6, 432 (2012).

${ }^{2}$ J. Faist, Quantum Cascade Lasers (Oxford University Press, Oxford, 2013).

${ }^{3}$ J. P. Commin, D. G. Revin, S. Y. Zhang, A. B. Krysa, K. Kennedy, and J. W. Cockburn, Appl. Phys. Lett. 97, 031108 (2010).

${ }^{4}$ G. Scalari, C. Walther, M. Fischer, R. Terazzi, H. Beere, D. Ritchie, and J. Faist, Laser Photonics Rev. 3, 45 (2009).

${ }^{5}$ S. Fathololoumi, E. Dupont, C. Chan, Z. Wasilewski, S. Laframboise, D. Ban, A. Mátyás, C. Jirauschek, Q. Hu, and H. C. Liu, Opt. Express 20, 3866 (2012).

${ }^{6}$ C. Sirtori, S. Barbieri, and R. Colombelli, Nat. Photonics 7, 691 (2013).

${ }^{7}$ M. A. Belkin and F. Capasso, Phys. Scr. 90, 118002 (2015).

${ }^{8}$ S. Khanal, L. Zhao, J. L. Reno, and S. Kumar, J. Opt. 16, 094001 (2014).

${ }^{9}$ S. Kumar, C. W. I. Chan, Q. Hu, and J. L. Reno, Appl. Phys. Lett. 95, 141110 (2009).

${ }^{10}$ G. Scalari, M. I. Amanti, C. Walther, R. Terazzi, M. Beck, and J. Faist, Opt. Express 18, 8043 (2010).

${ }^{11}$ G. Bastard, Wave Mechanics Applied to Semiconductor Heterostructures (EDP Science, Paris, 1990).

${ }^{12}$ C. Ndebeka-Bandou, A. Wacker, F. Carosella, R. Ferreira, and G. Bastard, Appl. Phys. Express 6, 094101 (2013).

${ }^{13}$ R. Nelander and A. Wacker, J. Appl. Phys. 106, 063115 (2009).

${ }^{14}$ D. Stehr, C. Metzner, M. Helm, T. Roch, and G. Strasser, Phys. Rev. Lett. 95, 257401 (2005).

${ }^{15}$ D. Stehr, M. Helm, C. Metzner, and M. C. Wanke, Phys. Rev. B 74, 085311 (2006).

${ }^{16}$ T. Antoni, M. Carras, X. Marcadet, B. Vinter, and V. Berger, Appl. Phys. Lett. 97, 042102 (2010).

${ }^{17}$ C. Ndebeka-Bandou, F. Carosella, R. Ferreira, A. Wacker, and G. Bastard, Appl. Phys. Lett. 101, 191104 (2012).

${ }^{18}$ C. Ndebeka-Bandou, F. Carosella, R. Ferreira, and G. Bastard, Appl. Phys. Lett. 102, 191105 (2013).

${ }^{19}$ C. Ndebeka-Bandou, F. Carosella, R. Ferreira, A. Wacker, and G. Bastard, Semicond. Sci. Technol. 29, 023001 (2014).

${ }^{20}$ J. I. Pankove, Optical Processes in Semiconductors (Dover, New-York, 1971).

${ }^{21}$ R. Ferreira and G. Bastard, Phys. Rev. B 40, 1074 (1989). 